MEI

II, vol. 5 $\mathrm{n}^{0} 9$

\title{
Conservación y preservación de documentos audiovisuales
}

\author{
Nelson Javier Pulido Daza \\ Universidad de La Salle, Bogotá, Colombia
}

Recibido el 22-11-2014

\section{Resumen}

Se exponen aspectos relacionados con la conservación y la preservación de documentos audiovisuales. El artículo se inicia con una descripción general de la Fundación Patrimonio Fílmico Colombiano, siendo ésta la institución encargada de la salvaguarda de los documentos fílmicos de la Nación. Se presenta como una herramienta que evidencia ciertas características a tener en cuenta en la elaboración de un Sistema de Conservación y Preservación con base en lineamientos nacionales e internaciones, o que permite fundamentar una estructura pertinente y orientada al contexto de la entidad y el Estado. Se analizan las estructuras de las Normas de descripción con el fin de establecer una integridad con lo estipulado en los Programas de Gestión Documental.

\section{Palabras clave}

Conservación, preservación, documentos audiovisuales, sistemas de conservación y preservación, Patrimonio audiovisual, Fundación Patrimonio Fílmico Colombiano, Colombia .

\section{Title}

Conservation and preservation of audiovisual documents

\begin{abstract}
Aspects related to the conservation and preservation of audiovisual documents are presented. The article begins with an overview of the Colombian Film Heritage Foundation being the institution responsible for safeguarding the film documentation of the Nation, is presented as a tool that evidences certain characteristics to consider in developing a Conservation and Preservation System based on national and international guidelines allowing to support a structureoriented relevance context of the entity; Rules of description structures are analyzed in order to establish an integrity with the provisions of Document Management Programs.
\end{abstract}

\section{Keywords}

Conservation, preservation, audiovisual documents, conservation and preservation systems, Audiovisual Heritage, Colombian Film Heritage Foundation, Colombia. 


\section{1.- Introducción}

Una de las herencias más destacadas, representativas y de mayor influencia en la sociedad es el Patrimonio Audiovisual. Las grabaciones con sonido y movimiento tienen la facultad de ser elementos que condensan la memoria y el legado del mundo; a través de éstos se pueden revivir diferentes épocas de la humanidad, ya que emergen como ventanas temporales que dan fe de las transformaciones ocurridas en el tiempo, además de ser elementos que pueden interactuar a nivel sensitivo con aquel que los consulta. Sus soportes contienen un valor indeterminado para la humanidad, es por ello que la UNESCO junto con el Consejo de Coordinación de las Asociaciones de Archivos Audiovisuales (CCAAA) han establecido el 27 de octubre como el Día Mundial del Patrimonio Audiovisual; hecho que les permite obtener recursos para la gestión y para los aspectos técnicos, sociales y financieros que amenazan la salvaguarda de los mismos (UNESCO, 2013). La debilidad de este tipo de documentos hace que se preste especial interés en su conservación y preservación puesto que son fuentes de carácter informativo, evidencial y testimonial para las generaciones emergentes.

En el ámbito colombiano, hace más de 25 años surgió como institución la Fundación Patrimonio Fílmico Colombiano, la cual es responsable de la restauración y la catalogación de varios fragmentos y piezas completas de la identidad y la historia colombiana. Un ejemplo del valor de estos documentos se encuentra en el título de la película documental "El Drama del quince de octubre" que tal vez no diga mucho, pero si se concibe como un documento perdido de carácter histórico en el cual de manera informativa se registró un magnicidio que retrasó de forma considerable el desarrollo del cine nacional, su sentido cambia.

Es precisamente esta manera de comprender los documentos audiovisuales la premisa del siguiente artículo, el cual tiene como objetivo principal establecer una herramienta que defina los lineamientos y las características propias de los conceptos de conservación y preservación orientada a los documentos audiovisuales tomando como base el marco normativo nacional e internacional. Para ello, se sustenta en dos objetivos específicos, los cuales son: en primer lugar, elaborar un concepto técnico, jurídico y comercial del material audiovisual que reposa en las instalaciones de la Fundación Patrimonio Fílmico; y en segundo lugar, presentar los principales factores, medios y elementos que se deben tener en cuenta para su correcta conservación. Lo anterior enfocado desde una perspectiva objetiva con el fin de establecer un mecanismo de concienciación sobre la importancia que estos documentos representan al ser integrantes de la denominada identidad nacional. 


\section{2.- Antecedentes contextuales}

Después de varios intentos que datan de los años cincuenta por conformar un archivo audiovisual en Colombia, éste sólo se instituyó a partir de 1986. Es así como el acervo fílmico nacional encuentra un sitio en el cual la recuperación, el almacenamiento y la custodia de más de noventa mil elementos clasificados entre rollos de cine, casetes de video y registros sonoros, tienen un lugar especializado para su salvaguarda como bienes de interés cultural.

La Fundación Patrimonio Fílmico Colombiano en colaboración con otras entidades ha promovido la producción de diferentes tipos de obras entre los que destacan obras audiovisuales, publicaciones $y$ exhibiciones que fundamentan y dan fe de la historia del cine colombiano; cabe destacar que dentro de su gestión han logrado estudiar y sistematizar medio millar de largometrajes filmados en el territorio nacional en casi noventa años de historia (Fundación Patrimonio Fílmico Colombiano, 2006, Universal, 2013).

"Con la Ley del Cine, el país pasó de filmar cuatro a veintitrés películas, como ocurrió en 2012" comentó la directora de la Fundación, Miriam Garzón de García, en una entrevista realizada en marzo de 2013. "Esta Ley 814 de 2003 ha permitido la producción de nuevas películas, el auge de nuevas iniciativas y el fortalecimiento de la convocatoria del Fondo para el Desarrollo Cinematográfico. De igual forma se debe mencionar que dentro de sus logros destacan la repatriación de algunas producciones que han sido guardadas en el exterior por motivos de censura política, como fue el caso de "Canaguaro", que registra la violencia ocurrida en los "Llanos Orientales" (Universal, 2013).

El recuperar esa memoria y reconstruir la historia del cine colombiano por medio de pequeñas piezas en movimiento hace que la gestión de la Fundación no pase desapercibida. Sus principales logros son:

- La recuperación, almacenamiento y custodia de un acervo de más de noventa mil elementos.

- La gestión para la restauración y preservación de los primeros registros cinematográficos colombianos.

- Ser poseedora y depositaria de bienes de interés cultural de la Nación gracias a la Resolución No 09663 de 2001 del Ministerio de Cultura.

- El restaurar y preservar un grupo de películas pertenecientes a las primeras épocas del cine nacional, junto con la restauración y preservación de las primeras obras del periodo del cine sonoro colombiano. 
- La realización de la Primera Muestra de Cine Mudo Colombiano.

- La salvaguarda, catalogación, indización y divulgación del Archivo Histórico Cinematográfico de los Acevedo, pioneros del periodismo audiovisual en Colombia.

- La promoción sobre la producción de obras audiovisuales, publicaciones y exhibiciones que muestran y fundamentan la historia del cine colombiano.

- El formalizar los procesos propios de los archivos audiovisuales tales como la búsqueda, recuperación, almacenamiento, inventario, verificación técnica, restauración editorial y catalogación.

- El proporcionar imágenes de archivo tanto para nuevas producciones nacionales e internacionales, como para otros programas.

- La realización y producción de Historias de la historia, como serie documental colombiana.

- La creación y puesta en servicio de una filmoteca y videoteca circulantes.

- La creación de un centro de documentación y una biblioteca especializados.

Finalmente, en colaboración con la Cinemateca del Caribe de Barranquilla y con la Cinemateca Distrital de Bogotá, se perfila como gestor principal del Sistema de Información del Patrimonio Audiovisual Colombiano, Sipac (Fundación Patrimonio Fílmico Colombiano, 2006).

\section{3.- Justificación normativa}

Desde el punto de vista internacional los lineamientos que se contemplan para los documentos audiovisuales se sustentan bajo las directrices resultantes de la $21^{a}$ reunión de la Conferencia General de la UNESCO (1980): Recomendación sobre la Salvaguardia y la Conservación de las Imágenes en Movimiento. Las mismas se presentan como una herramienta de utilidad enfocada en la gestión y la conservación teniendo en cuenta los problemas técnicos que plantean en la preservación del patrimonio. Otras organizaciones internacionales relacionadas con los documentos audiovisuales son el Consejo Internacional de Archivos (CIA), el Consejo Internacional del Cine y de la Televisión (CICT) y la Federación Internacional de Archivos de Televisión (FIAT). Es precisamente esta última la que ha establecido una serie de normas como guía, en base a 
criterios de carácter temporal, material y de autoridad que son aplicables a documentos de interés histórico, de interés sociológico, entre otros (Ronco López, 2009).

El siguiente es un listado de otras organizaciones que trabajan en paralelo a las anteriores:

- Asociación Internacional de Archivos Sonoros y audiovisuales (IASA) fundada en 1969 en Amsterdam (Holanda).

- Federación Internacional de Archivos de Filmes (FIAF) fundada en 1938 en París (Francia).

- Association of MovingImageArchivist (AMIA) fundada en 1960 (Estados Unidos).

- Federation of Commercial Audiovisual Libraries (FOCAL) fundada en 1985 en Londres (Inglaterra).

- EuropeanAudivisualObservatory fundado en 1992 en Estrasburgo (Francia).

- Memory Archives Programes (MAP-TV) fundada en 1989.

Ahora bien, la necesidad y el riesgo que presentan este tipo de documentos es su rápida obsolescencia. De hecho, según Linda Tadic ${ }^{2}$ dentro de 25 años la cinta de video habrá dejado de existir o de ser fabricada (Tadic, 2012); a lo que actualmente se realizan grandes esfuerzos puesto que este tipo de documentos carecen de estatutos, cartas fundacionales o documentos equivalentes que puntualicen tanto sus funciones como sus mecanismos de protección y finalmente su disposición. Este fenómeno los hace vulnerables a los cambios, lo que deriva en su definitiva pérdida (Edmondson, 2004).

\section{1- Marco Normativo Internacional}

\subsection{1- Instrumentos normativos}

En consonancia con los criterios establecidos por la FIAT, los documentos audiovisuales responden a algo denominado Criterio de Nacionalidad (relativo al vínculo jurídico y político de un individuo con el Estado) que articulado con los valores patrimoniales (aquello que es herencia y derecho de una persona natural o jurídica) representan el testimonio o la memoria de una Nación.

Contiguo a lo anterior la UNESCO propuso la fundación del Depósito Legal Nacional para las imágenes en movimiento. Esta denominación diferencia las películas de las imágenes fijas, incluyendo también los formatos propios del movimiento para poder proyectarlas(Ronco López, 2009). 
De acuerdo con dicha propuesta, las instituciones archivísticas internacionales buscan vincular este tipo de instituciones nacionales, con el fin de compartir su acervo documental de forma articulada por medio de Normas que interactúen entre sí.

A propósito de ello la Norma UNE-EN 15907 es una iniciativa europea que intenta definir un conjunto de metadatos enfocados en la descripción completa del material cinematográfico (Tadic, 2012). Su alcance pretende:

- Establecer un conjunto de metadatos para la descripción de las obras cinematográficas, así como una terminología para su uso por las partes que deseen intercambiar tales metadatos descriptivos.

- También define algunas entidades básicas y las relaciones útiles para la definición de modelos de datos, así como para estructurar representaciones jerárquicamente ordenadas y con número de serie de metadatos sobre las obras cinematográficas, incluidas sus variantes, manifestaciones e ítems.

Esta norma tiene la facultad de poderse utilizar como una lista de control de elementos y datos, gracias a su esquema le permite una buena interoperabilidad con diferentes bases de datos, además especifica el conjunto de metadatos y terminología para la descripción que deben utilizar las entidades que deseen intercambiar dichos metadatos descriptivos (Gómez Mascarell, 2012).

\subsection{2-Norma Internacional General de Descripción Archivística ISAD (G)} De acuerdo a la norma anterior desde la Archivística se plantea la Norma ISAD $(G)$ como una guía general para la elaboración de descripciones archivísticas, con la facultad de utilizarse en conjunto con las normas existes en cada país. De hecho, las normas de descripción archivística se basan en la descripción de lo general a lo particular, como consecuencia del principio de procedencia, lo que le permite presentar una estructura de aplicación general a entornos manuales o automatizados (CIA, 2000).

Sin embargo, cabe destacar que Antonia Heredia (1998) señala que después de un conjunto de ejemplos tomados como referencia en el ámbito de la administración local, encuentra ciertos elementos que deben ser evaluados, lo que lleva a entender la norma como "un formato universal, común -una guía general- para la descripción: es decir para la función, no para sus instrumentos". De igual forma establece que si la norma es algo deficiente para ser aplicada en el Archivo desde el punto de vista como institución, sin embargo, sí es aplicable al archivo como contenido documental. Así pues, es aplicable a los fondos y las colecciones del patrimonio documental. 
El progreso de la Norma ISAD $(\mathrm{G})$ por parte del CIA ha tratado de unificar diferentes conceptos existentes tanto internacional como nacionalmente, por lo que plantea su desarrollo en base de las normas nacionales. Antonia Heredia (1991) explica "normalizar la descripción no es inventar nada nuevo sino aprovechar y aplicar las reglas que tenemos, que, pese a todo, están más o menos consensuadas". La idea es normalizar pero que permanezcan intactos los criterios archivísticos, siendo los instrumentos equivalentes a los niveles de información.

La norma tiene reglas generales que pueden o tienen la facultad de aplicarse independientemente del tipo documental o del soporte físico de los documentos. Es en este momento es donde entra la descripción multinivel (comprendida como una técnica de análisis documental que consiste en describir un fondo y las partes que lo integran mediante elementos apropiados para cada nivel), relacionando las descripciones resultantes de manera jerárquica (Bonal Zazo, 2001).

Así pues, los niveles de la ISAD $(\mathrm{G})$ son los siguientes:

1. COLECCIÓN. (CORRESPONDE A LOS DOCUMENTOS DE CARÁCTER PERSONAL COMPRADOS Y/O DONADOS POR UNA PERSONA NATURAL).

2. FONDO.

3. SECCIÓN.

4. SERIE / SUBSERIE.

5. UNIDAD DOCUMENTAL COMPUESTA.

6. UNIDAD DOCUMENTAL SIMPLE.

En este orden de ideas es necesario resaltar que la Norma Internacional General de Descripción Archivística ISAD $(\mathrm{G})$ y la Norma ISAAR (CPF) son complementarias entre sí, lo que permite que la información de los diferentes niveles de descripción archivística esté contextualizada.

\subsection{3- Norma Internacional sobre Registros de Autoridad de Archivos relativos a Entidades, Personas y Familias ISAAR (CPF)}

Por otro lado, se encuentra la Norma Internacional sobre Registros de Autoridad de Archivos relativos a Entidades, Personas y Familias ISAAR $(\mathrm{CPF})$ la cual se establece como una guía para la elaboración de registros de autoridad, mediante encabezamientos archivísticos de autoridades, los cuales describen entidades, familias o personas que surgen como productores; esto permite controlar tanto la creación como el empleo de los puntos de acceso, lo que en conjunto establece ciertas similitudes descriptivas con los recursos bibliográficos y museográficos(CIA, 2004). 
Para explicar el alcance de esta norma es necesario definir qué es un registro de autoridad, el cual se constituye en la entrada autorizada de un nombre o un asunto en un catálogo. Este término es más familiar en áreas como la bibliotecología o la museología de donde "el establecimiento de una autoridad viene de un registro,el registro de autoridad, en el cual, a la forma normalizada del nombre se agrega la información que permitió su establecimiento y su asunción como una autoridad" (Marques de Fonseca, 2004).

Las ventajas de establecer los registros de autoridad tal como menciona Marques de Fonseca (2004) son:

- El acercamiento del trabajo con los profesionales de las bibliotecas y los museos.

- La elaboración de un nuevo tipo de recurso constituyendo una base de datos separada pero interconectada.

- El control de la creación y uso de puntos de acceso.

- La documentación de la relaciones de los diferentes productores de documentos.

Referencia Marques de Fonseca (2004) que estructuralmente la Norma se desenvuelve en cuatro áreas:

- ÁREA DE IDENTIFICACIÓN / ESTABLECE LAS FORMAS AUTORIZADAS DEL NOMBRE

- ÁREA DE LA DESCRIPCIÓN / INFORMA SOBRE LA HISTORIA, LAS ACTIVIDADES Y EL CONTEXTO DE LA ENTIDAD.

- ÁREA DE RELACIONES / REGISTRA Y DESCRIBE LAS RELACIONES ENTRE ESTA ENTIDAD Y OTRAS

- ÁREA DE CONTROL / INFORMA ACERCA DE LA CREACIÓN DEL REGISTRO DE AUTORIDAD

La institución de esta norma exige el desarrollo y el establecimiento de normas, reglas y políticas nacionales que mediante el debido proceso dará la posibilidad de integración de procesos con fondos y colecciones existentes en los archivos y otras unidades de información con acervos documentales de interés nacional.

\subsection{4- Norma Internacional para la Descripción de Funciones (ISDF)}

Relacionado a lo anterior se encuentra la Norma Internacional para la Descripción de Funciones (ISDF) la cual se perfila como una guía para la elaboración de descripciones de funciones de entidades relacionadas con la producción y la conservación de documentos. Esta descripción explica la 
procedencia de documentos desde el contexto del uso y la producción, es decir que sus descriptores controlan los puntos de acceso desde la perspectiva archivística (CIA, 2007).

Desde una perspectiva integral esta Norma presenta ciertos conceptos que deben ser analizados de forma objetiva ya que en ella se presentan axiomas que no conservan las mismas denominaciones que se plantean desde el contexto archivístico. Por ello, debe realizarse un análisis crítico antes de su implementación puesto que los cambios en términos, conceptos y en la forma en que se plantea esta estructura no se adecua de manera sencilla a cualquier entidad.

A continuación el esquema planteado por la ISDF (CIA, 2007):

\title{
I. ELEMENTOS DE DESCRIPCIÓN DE UNA FUNCIÓN ISDF
}

\author{
1. ÁREA DE IDENTIFICACIÓN \\ 2. ÁREA DE CONTEXTO \\ 3. ÁREA DE RELACIONES \\ 4. ÁREA DE CONTROL
}

\section{RELACIÓN DE LAS FUNCIONES CON LAS ORGANIZACIONES}

\author{
1. FORMA AUTORIZADA DE LA ORGANIZACIÓN / \\ INSTITUCIÓN Y TÍTULO DEL RECURSO ASOCIADO \\ 2. NATURALEZA DE LA RELACIÓN \\ 3. FECHAS DE LA RELACIÓN
}

\subsection{5- Norma Internacional para la Descripción de Instituciones que Custodian Fondos de Archivos (ISDIAH)}

La Norma Internacional para la Descripción de Instituciones que Custodian Fondos de Archivos (ISDIAH) emerge como una serie de reglas generales que normalizan la descripción de instituciones que conservan fondos de archivo. Provee directrices para la identificación y el contacto con instituciones que contengan fondos de archivo, mediante directorios o listas autorizadas lo que permite generar listas autorizadas interrelacionadas con bibliotecas y museos a nivel regional, nacional e internacional, creando de esta forma enlaces comunes al patrimonio cultural (CIA, 2008). 
Si se observa de manera objetiva la Norma tiene como objeto la descripción de cualquier institución que custodie documentos de archivo, de forma tal que presenta cierta globalización. Su estructura presenta seis áreas, de las cuales, las cinco primeras hacen referencia a la institución y la última reseña la descripción del área de control.

El siguiente es el esquema diseñado (CIA, 2008):

\section{AREA DE IDENTIFICACION}

1.1. Identificador

1.2. Forma ( $\mathrm{s}$ ) autorizadas del nombre

1.3. Formas paralela (s) del nombre

1.4. Otras forma (s) del nombre

1.5. Tipo de institución que conserva fondos de archivo

2. AREA DE CONTACTO

2.1. Localización y dirección (es)

2.2. Teléfono, fax, correo electrónico

2.3. Personas de contacto

3. AREA DE DESCRIPCIÓN

3.1. Historia de la institución que conserva los fondos de archivo

3.2. Contexto cultural y geográfico

3.3. Estructura administrativa

3.4. Gestión de documentos y política de ingresos

3.5. Edificio(s)

3.6. Fondos y otras colecciones custodiadas

3.7. Instrumentos descripción, guías y publicaciones

4. AREA DE ACCESO

4.1. Horarios de apertura

4.2. Condiciones y requisitos para el uso y el acceso

4.3. Accesibilidad

5. AREA DE SERVICIOS

5.1. Servicios de ayuda a la investigación

5.2. Servicios de reproducción

6. AREA DE CONTROL

6.1. Identificador de la descripción

6.2. Identificador de la institución

6.3. Reglas y/o convenciones

6.4. Estado de elaboración

6.5. Nivel de detalle

6.6. Fechas de creación, revisión o eliminación

6.7. Lengua (s) y escritura $(\mathrm{s})$

6.8. Fuentes

6.9. Notas de mantenimiento 


\subsection{Marco Normativo Nacional ${ }^{s}$}

Con respecto al panorama nacional colombiano se identifican las siguientes directrices o normas que sustentan los procesos y las disposiciones relacionadas directa e indirectamente con la conservación y preservación de los documentos audiovisuales.

En primer lugar existe la Ley Cine (2003) "Por la cual se dictan normas para el fomento de la actividad cinematográfica en Colombia". Esta ley incrementó de forma considerable el desarrollo y la producción del cine a nivel nacional. Y de igual forma posibilitó el aumento del acervo documental de la Fundación Patrimonio Fílmico Colombiano, siendo su objetivo:

Propiciar un desarrollo progresivo, armónico y equitativo de cinematografía nacional y, en general, promover la actividad cinematográfica en Colombia. Para la concreción de esta finalidad se adoptan medidas de fomento tendientes a posibilitar escenarios de retorno productivo entre los sectores integrantes de la industria de las imágenes en movimiento hacia su común actividad, (...) Por su carácter asociado directo al patrimonio cultural de la Nación y a la formación de identidad colectiva, la actividad cinematográfica es de interés social. Como tal es objeto de especial protección y contribuirá a su propio desarrollo industrial y artístico y a la protección cultural de la Nación (Ley $814,2003)$.

Ahora bien, desde el compendio de Políticas Culturales, existen lineamientos que se encuentran involucrados directamente con las garantías que debe tener el Estado para con el patrimonio de la Nación. Por ello, estas políticas se postulan como eje fundamental en la salvaguarda de la memoria nacional, teniendo en cuenta dos componentes: en primer lugar, el contenido informativo y en segundo lugar, el soporte en el que se consigna; esto entendiendo el patrimonio documental como un documento de cualquier tipo o como un grupo de documentos que conforman una colección, un fondo o unos archivos (AGN, 2005).

A continuación una recopilación legal o un corpus legisque expresa la institución de medidas en la gestión documental con respecto a garantizar la adecuada conservación y preservación de los documentos audiovisuales:

- ACUERDO 07 DE 1994 (JUNIO 29),Reglamento General de Archivos. ARTICULO $6^{\circ}$. Campo de aplicación. "El presente reglamento se aplicará en todos los archivos oficiales del orden nacional, departamental, distrital, municipal; de las entidades territoriales indígenas; de las entidades territoriales que se creen por Ley; de las divisiones administrativas y en los archivos históricos especiales". 
- ACUERDO 11 DE 1996 (Mayo 22), "Por el cual se establecen criterios de conservación y organización de documentos". ARTICULO $4^{\circ}$ "El procedimiento mencionado debe seguirse para todo material gráfico que se encuentre en los archivos, bien sea que este mezclado con la documentación textual o que se halle de manera independiente".

- Ley 397 de 1997 (agosto 7). Ley General de Cultura. "Por la cual se desarrollan los artículos 70, 71 y 72 y demás artículos concordantes de la Constitución Política y se dictan normas sobre patrimonio cultural, fomentos y estímulos a la cultura, se crea el Ministerio de la Cultura y se trasladan algunas dependencias".

- LEY 594 DE 2000 (julio 14), “Ley General de Archivos”.

Artículo 19. Soporte Documental. Las entidades del Estado podrán incorporar tecnologías de avanzada en la administración y conservación de sus archivos, empleando cualquier medio técnico, electrónico, informático, óptico o telemático, siempre y cuando cumplan con los siguientes requisitos:

\section{Organización archivística de los documentos.}

Realización de estudios técnicos para la adecuada decisión, teniendo en cuenta aspectos como la conservación física, las condiciones ambientales y operacionales, la seguridad, perdurabilidad y reproducción de la información contenida en estos soportes, así como el funcionamiento razonable del sistema.

- PARAGRAFO 1. Los documentos reproducidos por los citados medios, gozarán de la validez y eficacia del documento original, siempre que se cumplan los requisitos exigidos por las leyes procesales y se garantice la autenticidad, integridad e inalterabilidad de la información.

- PARAGRAFO 2. Los documentos originales que posean valores históricos no podrán ser destruidos, aun cuando hayan sido reproducidos y/o almacenados mediante cualquier medio.

Artículo 21. Programas de Gestión Documental. Las entidades públicas deberán elaborar programas de gestión de documentos, pudiendo contemplar el uso de nuevas tecnologías y soportes, en cuya aplicación deberán observarse los principios y procesos archivísticos. 
- PARAGRAFO: Los documentos emitidos por los citados medios gozarán de la validez y eficacia de un documento original, siempre que quede garantizada su autenticidad, su integridad y el cumplimiento de los requisitos exigidos por las leyes procesales.

Artículo 22. Procesos Archivísticos. La gestión de documentos dentro del concepto de Archivo Total, comprende procesos tales como la producción o recepción, la distribución, la consulta, la organización, la recuperación y la disposición final de los documentos.

Artículo 48. Conservación de documentos en nuevos soportes. El Archivo General de la Nación dará pautas y normas técnicas generales sobre conservación de archivos, incluyendo lo relativo a los documentos en nuevos soportes.

Artículo 49. Reproducción de documentos. El parágrafo del artículo 2 de la Ley 80 de 1989 quedará así: "En ningún caso los documentos de carácter histórico podrán ser destruidos, aunque hayan sido reproducidos por cualquier medio".

- Decreto 4124 de 2004 (diciembre 10).Por el que se reglamenta el Sistema Nacional de Archivos.

- Ley 1185 de 2008 (marzo 12), o Ley de Patrimonio. Reforma parcialmente de la Ley 397 de 1997 o General de Cultura y actualiza la definición de Patrimonio cultural. ARTÍCULO $1^{\circ}$. Modifíquese el artículo $4^{\circ}$ de la Ley 397 de 1997 el cual quedará, así: Artículo 4. Integración del patrimonio cultural de la Nación. El patrimonio cultural de la Nación está constituido por todos los bienes materiales, las manifestaciones inmateriales, los productos y las representaciones de la cultura que son expresión de la nacionalidad colombiana, tales como la lengua castellana, las lenguas y dialectos de las comunidades indígenas, negras y creoles, la tradición, el conocimiento ancestral, el paisaje cultural, las costumbres y los hábitos, así como los bienes materiales de naturaleza mueble e inmueble a los que se les atribuye, entre otros, especial interés histórico, artístico, científico, estético o simbólico en ámbitos como el plástico, arquitectónico, urbano, arqueológico, lingüístico, sonoro, musical, audiovisual, fílmico, testimonial, documental, literario, bibliográfico, museológico o antropológico.

- DECRETO 763 DE 2009 (marzo 10), Por el cual se reglamentan parcialmente las Leyes 814 de 2003 y 397 de 1997 modificada por medio 
de la Ley 1185 de 2008, en lo correspondiente al Patrimonio Cultural de la Nación de naturaleza material.

\section{4.- Conservación de material audiovisual}

Actualmente existe cierta ambigüedad con respecto a los términos de Conservación y Preservación puesto que en América existen dos corrientes enfocadas a la conservación. La corriente anglosajona las divide en dos: por un lado, la Preservación entendida como las medidas preventivas y de durabilidad de los documentos; y por el otro, la Conservación entendida como el establecimiento de medidas para la restauración de documentos. Mientras que la corriente Latina considera la Conservación como una única disciplina con dos vertientes específicas, es decir, la Conservación Preventiva o Preservación y la Restauración (Someillán López, 2006).

Amelia Gómez Fernández, citada por (Someillán López, 2006), entiende la Conservación como "todas aquellas acciones que tienden a evitar posibles daños futuros de un bien cultural, gracias al conocimiento previo y el control de los riesgos potenciales de deterioro".

El Diccionario de Terminología Archivística (1995) la define como el "conjunto de procedimientos y medidas destinadas a asegurar, por una parte, la preservación o prevención de posibles alteraciones físicas en los documentos, y, por otra, la restauración de éstos cuando la alteración se ha producido"; cabe destacar que la diferencia de la Conservación Permanente, la cual es definida como una "situación derivada de la fase de valoración que afecta a los documentos que han desarrollado valores secundarios y que en consecuencia no pueden ser eliminados".

En el caso nacional, la Ley General de Archivos, en el Título XI presenta la siguiente perspectiva:

- ARTículO 46. Los archivos de la Administración Pública deberán implementar un sistema integrado de conservación en cada una de las fases del ciclo vital de los documentos.

- ARTÍCULO 47. Los documentos de archivo, sean originales o copias, deberán elaborarse en soportes de comprobada durabilidad y calidad, de acuerdo con las normas nacionales o internacionales que para el efecto sean acogidas por el Archivo General de la Nación.

- PARÁGRAFO. Los documentos de archivo de conservación permanente podrán ser copiados en nuevos soportes. En tal caso, deberá preverse un 
programa de transferencia de información para garantizar la preservación y conservación de la misma.

Estas medidas se toman como una propuesta de protección a los documentos de cara al deterioro ocasionado por el uso o no uso, los daños resultantes de la gestión administrativa y el abandono al que son sometidos en algunas ocasiones.

En definitiva, se propone la conservación como:

"Un proceso archivístico en el cual se pretende asegurar la integridad tanto física como del soporte que contiene la información, es decir sus propiedades funcionales por medio del desarrollo de medidas administrativas, económicas y técnicas que con base en la preservación y la restauración, cumplan con el propósito final de estos, el cual es la trasmisión de información y conocimiento.”

\section{5.- Preservación de material audiovisual}

La definición otorgada por el Diccionario de Terminología Archivística (1995) hace referencia al "conjunto de procedimientos intelectuales y mecánicos destinados a asegurar la perdurabilidad de los documentos"; sin embargo, la definición más concreta la presentan el International Institute for Conservation Canadian Group y la Canadian Association of Professional Conservators, citados por Saint Laurent(1998):

"Todas las acciones que se toman para retardar el deterioro de, o para prevenir el daño a, la propiedad cultural. La preservación incluye el control del ambiente y de las condiciones de uso y puede incluir tratamiento, a objeto de poder mantener, dentro de lo posible, una propiedad cultural de manera permanente."

Ahora bien, en un sentido más amplio Ecured (2014) sostiene que son:

“Todas las actividades económicas y administrativas que incluyen el depósito y la instalación de los materiales, la formación del personal, los planes de acción, los métodos y técnicas referentes a la preservación de los materiales de archivos y bibliotecas y a la información contenida en ellos. En otras palabras, organización y 
programación de todos los aspectos y actividades relacionadas con la conservación de las colecciones en el sentido más amplio.”

Para ello existen básicamente tres cuestiones que se deben considerar cuando se manipulan y se almacenan grabaciones de sonido, como se indica en Saint Laurent(1998):

- Mantenerlas libres de cualquier depósito de partículas extrañas;

- Mantenerlas libres de cualquier presión que pueda causar deformación; y

- Almacenarlas en un ambiente estable y controlado.

\section{6.- Sistema de conservación y preservación de documentos audiovisuales} El objetivo del diseño de un Sistema de Conservación y Prevención de Documentos Audiovisuales es el de otorgar una estructura de soluciones integrales enfocadas a los documentos recibidos en la Fundación Patrimonio Fílmico Colombiano, para evitar al máximo posibles riesgos que puedan generar el deterioro o pérdida de la información, como consecuencia del soporte en donde se encuentra la misma.

El Sistema Integrado debe contemplarse desde la Ley General de Archivos que, como se mencionó anteriormente en el Título XI, concreta las estrategias y los procesos de Preservación y Conservación en concordancia con el Programa Gestión Documental (PGD), bajo el concepto general de Archivo Total.

Para ello, es necesario que el sistema se articule mediante una metodología, técnica y herramientas archivísticas centradas en el ciclo vital del documento. En consecuencia, es necesario plantear dicha metodología desde las necesidades propias de los documentos de la Fundación puesto que es preciso tener en cuenta factores internos y externos que son objeto del deterioro y que afectan a esta documentación.

Para diseñar el Sistema se debe contemplar los siguientes aspectos:

- Las características informacionales, técnicas y funcionales de la documentación

- El conocimiento de los factores de deterioro

- La normatividad que interviene de forma directa e indirecta con el tipo de información.

- Preservar la memoria histórica de la Entidad 
Además de ello, es necesario tener en cuenta los siguientes aspectos que intervienen de forma directa en el desarrollo del Sistema:

- Sensibilización del personal

- Migración de documentos

- Control de factores ambientales y biológicos

- Asepsia de áreas y documentos

- Consideración de uso y manipulación

- Supervisión y mantenimiento de instalaciones.

- Prevención y toma de medidas de emergencia

De igual forma, es necesario tener en cuenta como menciona Someillán López (2006)los factores existentes al interior del Sistema:

I. Factores de deterioro

- La naturaleza del material

- El ambiente en el que se guardan las colecciones

- El modo en que el material se manipula

- Los desastres naturales y causados por el hombre

II. Factores externos

- Ambientales

- Temperatura y humedad

- Luz

- Contaminantes atmosféricos

- Material particulado en superficie

- Bióticos

- Desastres

- Antropogénicos

III. Factores internos

- Estabilidad química y física

- Calidad del procesamiento

- Materias primas

IV. Políticas de conservación preventiva

- Las cuales apuntan hacia el qué y el porqué del sistema

V. Diagnóstico de conservación

- Dan la prioridad a las diferentes colecciones con el fin de destinar recursos a las tareas propias de la conservación preventiva. 


\section{7.- Recomendaciones para la conservación de documentos digitales y audiovisuales}

El desarrollo de un buen Sistema de conservación debe responder a las siguientes preguntas Saint Laurent(1998):

- ¿Cuál es la importancia del tema documentado por esta colección o publicación? ¿Será su mayor importancia de carácter local, estatal, regional, nacional o internacional?

- ¿Cómo se relaciona esta colección o publicación con otros fondos que documenten el mismo tema?

- ¿Cómo se relaciona con materiales sobre el mismo tema localizados en otros fondos?

- ¿Será la información que contiene única o genera duplicidad en otros registros, publicaciones o fuentes guardadas en otros fondos?

- ¿Está comprometida la institución o la entidad en seguir documentando este tema?

- ¿Por qué es mejor invertir recursos en la preservación de este material en lugar de adquirir material nuevo?

- ¿Qué impacto tendrá la destrucción de este material en la documentación y comprensión del tema?

- Una vez analizado y determinado el nivel de importancia de cada documento se deben establecer las maneras o formas correctas para estructurar el Sistema y que éste responda a las necesidades de cada soporte en el que se encuentra la información.

Una vez analizado y determinado el nivel de importancia de cada documento se deben establecer las maneras o formas correctas para estructurar el Sistema y que éste responda a las necesidades de cada soporte en el que se encuentra la información.

\section{1- Documentos electrónicos}

Teniendo presente que los documentos electrónicos son "la información generada, enviada, recibida, almacenada o comunicada por medios electrónicos, ópticos o similares" (Ley 527, 2009), en los archivos las colecciones y los documentos electrónicos/digitales ${ }^{4}$ establecen cientos criterios fundamentales que se deben tener en cuenta.

\subsection{1- Características generales de los documentos electrónicos}

La adherencia a los estándares internacionales es un requisito importante para la disponibilidad y la legibilidad a largo plazo de cualquier documento 
electrónico. Los formatos admitidos contemplan los siguientes aspectos según señala el Programa de Gobierno en Línea del Ministerio de Tecnologías de la Información y las Comunicaciones (2009):

- Imagen y/o texto

- Cartografía vectorial y sistemas de información geográfica

- Compresión de ficheros

- Contenedores multimedia

- Sonido

- Video

Ahora bien, los formatos a tener en cuenta actualmente son los siguientes para la elaboración y creación del documento electrónico:

\begin{tabular}{|c|c|c|c|c|}
\hline Escenario & \multicolumn{4}{|c|}{ Formatos } \\
\hline \multirow{9}{*}{$\begin{array}{l}\text { Documentos presenta- } \\
\text { dos por la ciudadanía }\end{array}$} & \multicolumn{4}{|c|}{ Texto } \\
\hline & $\begin{array}{l}\text { TXT } \\
\text { RTF } \\
\text { ODF }\end{array}$ & $\begin{array}{l}\text { PDF } \\
\text { PDF/A } \\
\text { XML }\end{array}$ & $\begin{array}{l}\text { HTML } 4.01 / \\
\text { XHTML } 1.0 \\
\text { CSV }\end{array}$ & $\begin{array}{l}\text { OOXNL } \\
\text { MS Office }\end{array}$ \\
\hline & \multicolumn{4}{|c|}{ Imagen } \\
\hline & $\begin{array}{l}\text { JPG/JPEG } \\
\text { PDF }\end{array}$ & $\begin{array}{l}\text { PDF/A } \\
\text { PNG }\end{array}$ & TIFF & \\
\hline & \multicolumn{4}{|c|}{ Audio } \\
\hline & AAC & MP3 & Ogg/Oga & \\
\hline & \multicolumn{4}{|c|}{ Video y Contenedor } \\
\hline & $\begin{array}{l}\text { MPEG-4 } \\
\text { H.264 AVC } \\
\text { Ogg/Ogv }\end{array}$ & $\begin{array}{l}\text { WMV } \\
\text { AVI } \\
\text { OGM }\end{array}$ & $\begin{array}{l}\text { MKV } \\
\text { MP4 } \\
\text { ASF }\end{array}$ & $\begin{array}{l}\text { FLV } \\
\text { Quick Time } \\
\text { RealMedia }\end{array}$ \\
\hline & \multicolumn{4}{|c|}{ Texto } \\
\hline \multirow{7}{*}{$\begin{array}{l}\text { Documentos genera- } \\
\text { dos por las entidades }\end{array}$} & $\begin{array}{l}\text { TXT } \\
\text { RTF } \\
\text { ODF }\end{array}$ & $\begin{array}{l}\text { PDF } \\
\text { PDF/A } \\
\text { XML }\end{array}$ & $\begin{array}{l}\text { HTML 4.01/ } \\
\text { XHTML } 1.0\end{array}$ & $\begin{array}{l}\text { OOXNL } \\
\text { MS Office }\end{array}$ \\
\hline & \multicolumn{4}{|c|}{ Imagen } \\
\hline & $\begin{array}{l}\text { JPG/JPEG } \\
\text { PDF }\end{array}$ & $\begin{array}{l}\text { PDF/A } \\
\text { PNG }\end{array}$ & TIFF & \\
\hline & \multicolumn{4}{|c|}{ Audio } \\
\hline & AAC & MP3 & Ogg/Oga & \\
\hline & \multicolumn{4}{|c|}{ Video y Contenedor } \\
\hline & $\begin{array}{l}\text { MPEG-4 } \\
\text { H.264 AVC }\end{array}$ & $\begin{array}{l}\text { Ogg/Ogv } \\
\text { AVI }\end{array}$ & $\begin{array}{l}\text { OGM } \\
\text { MKV }\end{array}$ & $\begin{array}{l}\text { Mp4 } \\
\text { FLV }\end{array}$ \\
\hline $\begin{array}{l}\text { Documentos enviados } \\
\text { a la ciudadanía }\end{array}$ & \multicolumn{4}{|l|}{ PDF/A } \\
\hline $\begin{array}{l}\text { Documentos almace- } \\
\text { nados en el SGDEA }\end{array}$ & \multicolumn{4}{|c|}{$\begin{array}{l}\text { Los documentos deberían guardarse en el formato en que se reciben y gene- } \\
\text { ran. } \\
\text { Se recomienda utilizar PDF/A como formato de conservación a largo plazo }\end{array}$} \\
\hline
\end{tabular}

Fuente: Ministerio de Tecnologías de la Información y las Comunicaciones (2009) 
La obsolescencia tecnológica es un elemento que afecta al hardware y al software, por tanto, debe preverse y garantizar la conservación, acceso, lectura e integridad de los documentos sin importar el formato en que fueron producidos. Para asegurar la preservación de la información, el formato elegido debe ser legible por una aplicación durante el mayor tiempo posible; en tal caso se puede utilizar el formato PDF que actualmente se encuentra en estudio por parte de la ISO.

Tal como lo declara Cruz Mundet (2003) los requisitos para la organización de estos documentos se establecen desde tres fases, las cuales son:

1. Fase de diseño: se trata de intervenir en el diseño de los documentos y de los procesos en los que se insertan para garantizar la incorporación de requisitos archivísticos, entendidos como:

- Fiabilidad (contenido fidedigno).

- Autenticidad (inmunes a todo tipo de manipulación y alteración).

- Integridad (completos en su estructura y contenido).

- Accesibilidad (recuperables).

- Descripción (comprenden el contenido, el proceso y el contexto).

- Selección (evaluación incorporada).

2. Fase de utilización: en la que los documentos fiables, auténticos, íntegros y accesibles deben conservar estas características inalteradas mediante fórmulas de autentificación, para ello son necesarios otros requisitos archivísticos, primordialmente:

- Interconexión (documentos en otros lugares correspondan al procedimiento).

- Interrelación (mediante la clasificación e integración en sistemas híbridos).

- Transferencia (servidores destinados a la conservación perpetua).

3. Fase de conservación: se les mantiene en la misma forma y estado de transmisión en el que han sido creados, recibidos o guardados, mediante la renovación constante y la migración periódica:

- La renovación (reproducción completa).

- La migración (transferencia periódica entre configuraciones o entre 
generaciones de tecnología)

- La conservación (procedimientos de control).

La moderación de la temperatura y la humedad son dos acciones que se sabe alargan la vida útil de muchos soportes de almacenamiento, pero la conservación de soportes debe incluir:

- Suficiente capacidad de almacenamiento. Es importante tener en cuenta que el sistema debe tener espacio suficiente para almacenar los datos previstos durante todo el ciclo de vida de los mismos.

- El sistema debe ser capaz de duplicar los datos a medida que sea requerido sin pérdida de información y manteniendo la consistencia e integridad de los documentos, así como transferir los datos a un nuevo soporte con las mismas condiciones.

- Las opciones disponibles en este momento de soportes a largo plazo incluyen:

- Disco magnético: permite un acceso aleatorio a los datos, con posibilidad de modificarlos y una capacidad de almacenamiento superior a los $200 \mathrm{~Gb}$ y en constante incremento. Su vida útil estimada se sitúa en torno a los cinco años.

- Cinta magnética: el acceso a los datos es lineal con lo cual es más lenta la búsqueda y localización de los mismos. En general no permite modificación de los datos, sino la reescritura de los mismos. La vida útil y la capacidad de almacenamiento es similar a los discos magnéticos.

- Discos ópticos (CD y DVD): permiten un acceso aleatorio y en algunos casos la modificación de los datos. Su vida útil es mayor que en los casos anteriores variando desde los cinco a diez años de los productos con menos calidad a varias décadas para los de mayor calidad.

- Mantenimiento, soporte y programas de reemplazo. Los componentes del sistema deben ser reemplazados cíclicamente cada dos años. Los elementos de hardware normalmente tienen una vida útil de en torno a los cinco años antes de que el soporte técnico pueda ser difícil de obtener. Los soportes de almacenamiento también necesitan una actualización regular (reescribir los datos) y un reemplazo periódico por nuevos soportes.

- Transferir los datos a nuevos soportes de forma periódica. Los sistemas de almacenamiento descansan en una segura y completa réplica de los datos más que en alargar la vida útil de los soportes. Los datos deben ser copiados de soporte a soporte para evitar el impacto del deterioro de los materiales. A medida que nuevos tipos de soportes prueban su utilidad para el almacenamiento, los datos deben ser transferidos desde los soportes más 
antiguos. Esto debe realizarse antes de que los componentes de hardware o software necesarios para acceder a los datos sean retirados.

- Establecer condiciones de almacenamiento y manejo apropiadas. Los soportes de almacenamiento digital deben ser almacenados en condiciones que no aceleren su ritmo de deterioro natural. El principal riesgo para los soportes es la excesiva humedad y temperatura, suciedad u otras particularidades que puedan entorpecer el acceso a los datos, y en el caso de soportes ópticos, la luz que puede dañar los datos inscritos. Las cintas magnéticas en la actualidad están protegidas de tal forma que el borrado accidental debido a su exposición a un campo magnético no suele ser un grave problema. Tales cintas pueden ser utilizadas como sistema de almacenamiento digital. Normalmente se las dispondrá en una sala acondicionada con una temperatura controlada y una humedad relativa establecidas entorno a los 18 grados centígrados y 40\% respectivamente, un flujo continuo de aire limpio con una limpieza diaria que evite la acumulación de polvo y basura. Estas condiciones no deben fluctuar más de dos grados y un $10 \%$ de humedad relativa en un período de 24 horas. Los soportes ópticos tales como los CD deben ser almacenados en unas condiciones similares, incluyendo además una estancia semioscura para limitar su sensibilidad a la luz. Si bien se ha afirmado que las temperaturas extremadamente bajas, en torno a los cero grados, pueden ser perjudiciales para los soportes, esto no ha sido demostrado.

- Por otro lado, también pueden ayudar otros factores relativos al control del medio ambiente en que se almacena los objetos. Como básicos podríamos señalar:

- Mantener una temperatura en torno a los $20^{\circ} \mathrm{C}$.

- Mantener la humedad relativa en torno al 40\%.

- Evitar las oscilaciones rápidas o prolongadas de temperatura y humedad.

- Evitar la exposición a campos magnéticos y a fuentes de humos.

- Prohibir la comida y/o bebida en lugares de almacenamiento, así como el fumar.

- Almacenar los medios de forma vertical.

- Utilizar siempre las cajas originales.

- No abrir las cajas que protegen las cintas.

- Evitar la suciedad.

- Mantener los medios en sus cajas excepto cuando se estén usando.

- No tocar la superficie de los soportes, por ejemplo la cara grabable de los CD.

- Los CD deberían marcarse sólo en la parte superior y utilizando marcadores adecuados. 
- No dejar los soportes en los aparatos de visualización cuando no se estén usando.

- Políticas de redundancia y copias de seguridad: la importancia de la duplicación y las copias de seguridad no pueden ser desdeñadas, son fundamentales en todos los programas de conservación como un seguro básico contra el deterioro o pérdida si sólo existiera una única copia. Si bien almacenar múltiples copias de los mismos datos ofrece alguna protección contra los fallos, los programas de conservación deben también considerar el riesgo de una situación catastrófica tal como incendios, inundaciones, etc., que pueda dañar todas las copias existentes en un mismo lugar. Almacenar copias en diferentes lugares es un requisito básico.

- Planificación contra los posibles desastres: deben diseñarse planes de recuperación de datos tras una situación de fallo que son estándares en las tecnologías de la información. Los planes deben incluir situaciones hipotéticas de recuperación de datos a partir de soportes dañados, pero la recuperación de datos es costosa e incierta y debería ser vista como una alternativa poco satisfactoria a la correcta recuperación a partir de copias de seguridad.

\subsubsection{Otras clases de documentos electrónicos}

\begin{tabular}{|c|c|c|}
\hline Tipos & Descripción & Recomendación \\
\hline Bases de datos & $\begin{array}{l}\text { Colección datos afines, relacionados entre } \\
\text { sí y estructurados de forma tal que permi- } \\
\text { ten el acceso, manipulación y extracción } \\
\text { de ciertos subconjuntos de esos datos por } \\
\text { parte de programas creados para tal efecto } \\
\text { o lenguajes de búsqueda rápida. }\end{array}$ & $\begin{array}{l}\text { Establecer cortes de información para } \\
\text { seleccionar de ellos la que deba conser- } \\
\text { varse a largo plazo o de forma permanen- } \\
\text { te y transferirlos al Sistema de Documen- } \\
\text { tos Electrónicos de Archivo -SGDA-. Los } \\
\text { criterios a seguir para el establecimiento } \\
\text { de los cortes varían de acuerdo al conte- } \\
\text { nido de la base de datos y de la informa- } \\
\text { ción que deba conservarse. }\end{array}$ \\
\hline Páginas web & $\begin{array}{l}\text { Una página web está compuesta principal- } \\
\text { mente por información (texto y/o módulos } \\
\text { multimedia), así como por hipervínculos. } \\
\text { Además, puede contener o asociar datos } \\
\text { sobre el estilo que debe tener y como debe } \\
\text { visualizarse y también aplicaciones } \\
\text { "embebidas" con las que se puede interac- } \\
\text { tuar para hacerlas dinámicas. }\end{array}$ & $\begin{array}{l}\text { Deben definirse políticas para establecer } \\
\text { el tipo de contenido que se publica, la } \\
\text { periodicidad de su actualización, y el } \\
\text { responsable de producir y aprobar la } \\
\text { publicación. } \\
\text { Así mismo, se debe tener en cuenta la } \\
\text { conservación de la información, datos y } \\
\text { documentos de archivo publicado, de } \\
\text { forma que se cuente con un historial de } \\
\text { publicaciones en el sitio web. }\end{array}$ \\
\hline
\end{tabular}

Fuente: Ministerio de Tecnologías de la Información y las Comunicaciones (2009) 


\section{2- Documentos audiovisuales}

Las colecciones de audio y video requieren de un cuidado y manejo específico. De igual forma, es necesario destinar de un ambiente de almacenamiento específico en caso de que si información deba preservarse por más de diez años.

\subsection{1- Problemas generales con el medio magnético}

- Degradación del aglutinante: responsable de mantener unidas las partículas magnéticas en la cinta además de facilitar el transporte de la misma. Su humedad en interiores deberá ser menor de 10 a $20 \%$ HR. En caso de presentarse el síndrome de cinta pegajosa se debe tratar a una temperatura de $122^{\circ} \mathrm{F}\left(50^{\circ} \mathrm{C}\right)$ por tres días, tiempo en el cual la capa aglutinante se habrá reafirmado para volverse a usar. Es necesario tener en cuenta que los niveles de lubricante decrecen a medida que pasa el tiempo, como también se consumen cada vez que el documento es objeto de consulta.

- Inestabilidad de la partícula magnética: conocida también como pigmento, es la responsable del almacenamiento magnético de la información que se encuentra registrada, lo que hace mediante los cambios en la dirección del magnetismo. El fenómeno de inestabilidad presenta lo siguiente: la remanencia magnética, que consiste en la habilidad del pigmento en retener el campo magnético; la disminución de la misma genera con el tiempo una reducción importante de la señal de salida y por ende, pérdida de la información. La coercitividad, definida como la habilidad del pigmento en resistir la desmagnetización causada, por ejemplo, por un imán; la carencia de ésta hace que se genere perdida de la señal.

- Deformación del sustrato: es el encargado de dar a la cinta el apoyo necesario para ser transportada a través del grabador, siendo el poliéster su principal elemento, el cual ha mostrado ser estable en el tiempo. Las películas elaboradas en poliéster son altamente resistentes a la oxidación y ala hidrólisis. En condiciones de archivo es más resistente que el polímero aglutinante. Sin embargo, la excesiva presión del rollo de cinta, el envejecimiento y la mala calidad del bobinado pueden dar como resultado el fallo de lectura en el momento de ser reproducida. Para que esto no ocurra, lo mejor es almacenar el documento en un ambiente cuya temperatura y humedad no varíen demasiado, puesto que cada vez que hay un cambio significativo el rollo de cinta se contrae o se expande, modificándose las dimensiones en el soporte, lo que generalmente produce 
un fallo de lectura.

- Aspectos relativos al formato: de acuerdo a lo anterior, el cambio en las dimensiones del formato significa perdida de información. La cinta de video usa un formato de grabación de barrido helicoidal, lo que la hace más sensible a los cambios dimensionales; en contrate, la cinta de audio analógica utiliza la grabación longitudinal. La disposición de la cinta puede generar pérdida de lectura y doblez en la cinta en el momento de reproducirla, como también curvatura en la cinta, de modo que el cabezal de reproducción deforme la cinta al pasar por éste.

- Grabadores de cinta magnética: los dispositivos de grabación deben mantenerse en excelentes condiciones, puesto que aquellos que se encuentren sucios pueden arruinar las cintas distribuyendo la suciedad sobre su superficie, lo que también raya las mismas. En caso de estar alineados eléctricamente de forma inadecuada pueden causar problemas de señal y dar como resultado una reproducción de mala calidad.

\subsection{2- Evitar pérdida de información}

Las causas de perdida de información son:

- Puntos de deterioro de la cinta

- Robo

- Destrucción / fuego / agua / longevidad

El uso de copias múltiples como estrategia para evitar la pérdida de información resulta viable si son de consulta constante. De igual forma es aconsejable guardarlas en diferentes sitios. Si se dispone de fondos es preferible mantener el almacenamiento de acceso y el almacenamiento de archivo de la información más importante. De acuerdo con lo anterior, el ambiente de acceso mantiene la grabación disponible en todo momento para su uso. Si no es posible tener duplicado de la información es necesario limitar su acceso. Si la información es considerada muy valiosa se deben mantenerse varias copias junto con el original.

\subsection{3- Duración de los medios magnéticos}

La documentación al respecto es escasa. Si bien el método para determinar la duración aún no se ha establecido, sin embargo, existen ensayos de 
envejecimiento acelerado, en ambiente de temperatura / humedad y corrosión de manera controlada, que indican medir el rendimiento en el tiempo, permitiendo de esta forma desarrollar modelos que dejan predecir la vida útil junto con sus medios a largo plazo. Ahora bien, de acuerdo con datos proporcionados por fabricantes y publicaciones técnicas el límite máximo es de treinta años, pero hay que tener en cuenta que la Scientific American en una carta publicada en 1995 estimó la vida útil como mínino de 10 a 20 años.

\subsection{4- Evitar la degradación prematura}

Además de controlar la consulta y el almacenamiento es necesario tener en cuenta otros factores que intervienen en la degradación del material, es decir, que se debe tener en cuenta lo siguiente:

- El cuidado con la manipulación y el transporte.

- La calidad de las condiciones con las que se almacena.

- El número de veces que se usa la cinta.

- Los componentes físicos que forman parte de su estructura.

- Variación de la calidad del fabricante.

- La futura disponibilidad de la tecnología o del sistema de acceso.

Este último punto es de especial cuidado puesto que existen cintas en muy buen estado pero los equipos necesarios para su reproducción no se encuentran o no funcionan correctamente.

\subsection{5- Cuidado y manipulación}

Para ello se plantea las siguientes recomendaciones:

- Almacenar y usar carretes en un ambiente limpio.

- Evitar la contaminación de polvo, huellas dactilares, comida, humo, cenizas de cigarrillo y contaminantes aerotransportados.

- No dejarlos caer.

- Protección de la luz solar fuerte y evitar el contacto con agua o sustancias solubles.

- No almacenarlas en sitos de calefacción alta, umbrales de ventanas, televisores, equipos electrónicos u otras máquinas que puedan establecer cargas iónicas. 
- Cuando las cintas no estén en uso deben estar almacenadas sobre una superficie mayor, sobre todo si es por un largo periodo de tiempo.

\subsection{6- Condiciones y normas de almacenamiento}

Además de conservarse en un ambiente limpio, la temperatura y la humedad establecen ciertos riesgos en los componentes físicos en la vida útil de la cinta. En algunos casos las temperaturas inferiores a $32^{\circ} \mathrm{F}\left(0^{\circ} \mathrm{C}\right)$ pueden acortar de forma dramática la vida de la cinta, como consecuencia de una riesgosa exudación de lubricante. Los cambios bruscos de temperatura introducen presión en el rollo. En caso de realizarse una reproducción en un lugar diferente al del almacenamiento es necesario dejar aclimatar la cinta a la nueva temperatura.

\subsection{7- Recomendaciones de almacenamiento}

A continuación se adjuntan tablas elaboradas por entidades técnicas que sugieren recomendaciones generales para la conservación de la documentación en los archivos.

Tabla1. Recomendaciones actuales para el almacenamiento de cintas magnéticas. Fuente: Saint Laurent

\begin{tabular}{|c|c|c|c|}
\hline Agencia/Investigador & Fecha & Temperatura & Humedad relativa \\
\hline Cuddihy & 1982 & $\begin{array}{l}65^{\circ} \mathrm{F}+/-3^{\circ} \mathrm{F} \\
18^{\circ} \mathrm{C}+/-2^{\circ} \mathrm{C}\end{array}$ & $40 \%+/-5 \%$ \\
\hline SMPTE (RP-103) & 1982 & $\begin{array}{l}70^{\circ} \mathrm{F}+/-4^{\circ} \mathrm{F} \\
21^{\circ} \mathrm{C}+/-2^{\circ} \mathrm{C}\end{array}$ & $50 \%+/-20 \%$ \\
\hline NARA & 1990 & $\begin{array}{l}65^{\circ} \mathrm{F}+/-3^{\circ} \mathrm{F} \\
18^{\circ} \mathrm{C}+/-2^{\circ} \mathrm{C}\end{array}$ & $40 \%+/-5 \%$ \\
\hline
\end{tabular}


MEI, II, Vol. 5, no 9, pág. 148

Tabla 2. Aspectos clave del almacenamiento, acceso y archivo de las cintas magnéticas. Fuente: Saint Laurent (1998)

\begin{tabular}{|c|c|c|}
\hline Característica clave & Almacenamiento de acceso & Almacenamiento de archivo \\
\hline Función & $\begin{array}{l}\text { Proporcionar un almacenamien- } \\
\text { to de medios que permita un } \\
\text { acceso y reproducción inmedia- } \\
\text { tos }\end{array}$ & $\begin{array}{l}\text { Proporcionar almacenamiento que } \\
\text { preserve los medios por el mayor } \\
\text { tiempo posible }\end{array}$ \\
\hline $\begin{array}{l}\text { ¿Se requiere aclimatación antes } \\
\text { de la reproducción? }\end{array}$ & No & Sí \\
\hline Esperanza de vida de los medios & $\begin{array}{l}\text { Por lo menos } 10 \text { años cuando se } \\
\text { almacena en las condiciones de } \\
\text { temperatura y humedad indica- } \\
\text { das }\end{array}$ & $\begin{array}{l}\text { La máxima permitida para el tipo } \\
\text { de medio en particular }\end{array}$ \\
\hline Valor de ajuste de la temperatura & $\begin{array}{c}\text { Próxima o igual a la temperatura } \\
\text { ambiental interna. En el rango: } \\
60^{\circ} \text { a } 74^{\circ} \mathrm{F}\left(15^{\circ} \text { a } 23^{\circ} \mathrm{C}\right)\end{array}$ & $\begin{array}{c}\text { Significativamente inferior a la } \\
\text { del ambiente interno. Tan baja } \\
\text { como } 40^{\circ} \mathrm{F}\left(5^{\circ} \mathrm{C}\right)\end{array}$ \\
\hline Valor de ajuste de la humedad & $\begin{array}{c}\text { La del ambiente o cerca de este } \\
\text { nivel: En el rango de: } 25 \text { a } 55 \% \\
\text { HR }\end{array}$ & $\begin{array}{l}\text { Significativamente inferior a la } \\
\text { temperatura ambiente. Tan baja } \\
\text { como } 20 \% \text { de HR }\end{array}$ \\
\hline Variaciones de temperatura & $\begin{array}{l}\text { La diferencia entre el valor } \\
\text { máximo y el mínimo no debería } \\
\text { exceder los } 7^{\circ} \mathrm{F}\left(4^{\circ} \mathrm{C}\right)\end{array}$ & $\begin{array}{l}\text { La diferencia entre el valor máxi- } \\
\text { mo y el mínimo no debería exce- } \\
\text { der los } 7^{\circ} \mathrm{F}\left(4^{\circ} \mathrm{C}\right)\end{array}$ \\
\hline Variaciones de humedad & $\begin{array}{l}\text { La diferencia entre el valor } \\
\text { máximo y el mínimo no debería } \\
\text { exceder el } 20 \% \text { de HR }\end{array}$ & $\begin{array}{l}\text { La diferencia entre el valor máxi- } \\
\text { mo y el mínimo no debería exce- } \\
\text { der el } 10 \% \text { de HR }\end{array}$ \\
\hline
\end{tabular}

Tabla 3. Tiempos de aclimatación para medios magnéticos retirados del almacenamiento de archivo. Fuente:Saint Laurent (1998)

\begin{tabular}{|l|c|c|}
\hline \multicolumn{1}{|c|}{ Formato de cinta } & $\begin{array}{c}\text { Tiempo para la aclima- } \\
\text { tación a la temperatura } \\
\text { (horas) }\end{array}$ & $\begin{array}{c}\text { Tiempo para la acli- } \\
\text { matación a la hume- } \\
\text { dad (días) }\end{array}$ \\
\hline Casete de audio compacto & 1 & 6 \\
\hline Cintas de carrete de $1 / 4$ pulgada & 1 & 1 \\
\hline Cintas de carrete de 2 pulgadas & 16 & 50 \\
\hline Cartucho VHS/Beta & 2 & 4 \\
\hline Casete de video de $8 \mathrm{~mm}$ & 1 & 2 \\
\hline Casete U matic-4-8 & 4 & 8 \\
\hline
\end{tabular}




\section{8.- Conclusiones}

La importancia que genera la conservación de los documentos audiovisuales con respecto al patrimonio cultural de la Nación, es de carácter fundamental puesto que en ellos se condensan características lingüísticas, artes representativas, sucesos históricos, entre otros contextos que enriquecen la memoria del país y de la humanidad.

Las entidades encargadas de preservar, restaurar y hacer accesibles los documentos audiovisuales como patrimonio nacional deben establecer mecanismos que permitan el desarrollo del intelecto, como recursos educativos, históricos y culturales de carácter importante. Es por ello necesario dirigir recursos y establecer estructuras metodológicas efectivas para la plena conservación de los mismos. De igual forma, es necesario instaurar la articulación de diferentes normas que ofrezcan alternativas interdisciplinarias, lo que permite utilizar este acervo documental por otras instituciones.

Si bien es cierto que existen ciertas divergencias entre las mismas normas, es virtud de su aplicación el subsanar dichos inconvenientes, estableciendo un lenguaje unilateral por medio del fomento de la cooperación entre organizaciones. Dicha iniciativa permite la exhibición y el uso de este tipo de materiales.

El desarrollo de una metodología sustentada en un Programa de Conservación y Preservación desde los marcos legislativos nacional e internacional otorga a la institución la posibilidad de interactuar con otras entidades siendo ésta un artefacto y una fuerza para el cambio y el desarrollo social.

Finalmente, la Fundación Patrimonio Fílmico Colombiano emerge como una institución encargada del fomento de la investigación, la educación y el desarrollo nacional, que tiene encomendada la conservación y la preservación de nuestro patrimonio cultural. Dado que su acervo documental contiene la información resultante de la tradición oral y visual, se le asigna una responsabilidad preponderante con respecto al acceso a estos documentos por las nuevas generaciones.

La adherencia a los estándares internacionales es un requisito importante para la disponibilidad y la legibilidad a largo plazo de cualquier documento electrónico.

La obsolescencia tecnológica es un elemento que afecta a hardware y software, por tanto, debe preverse y garantizar la conservación, acceso, lectura 
e integridad de los documentos sin importar el formato en que fueron producidos.

Para asegurar la preservación de la información, el formato elegido debe ser legible por una aplicación durante el mayor tiempo posible.

La moderación de la temperatura y la humedad son dos acciones que se sabe alargan la vida útil de muchos soportes de almacenamiento, pero la conservación de soportes debe incluir:

- Suficiente capacidad de almacenamiento. Es importante tener en cuenta que el sistema debe tener espacio suficiente para almacenar los datos previstos durante todo el ciclo de vida de los mismos.

- El sistema debe ser capaz de duplicar los datos a medida que sea requerido, sin pérdida de información y manteniendo la consistencia e integridad de los documentos, así como transferir los datos a un nuevo soporte con las mismas condiciones.

Las opciones disponibles en este momento de soportes a largo plazo incluyen:

- Disco magnético: permite un acceso aleatorio a los datos, con posibilidad de modificarlos y una capacidad de almacenamiento superior a los $200 \mathrm{~Gb}$ y en constante incremento. Su vida útil estimada se sitúa en torno a los cinco años.

- Cinta magnética: el acceso a los datos es lineal con lo cual es más lenta la búsqueda y localización de los mismos. En general no permite modificación de los datos, sino la reescritura de los mismos. La vida útil y la capacidad de almacenamiento es similar a los discos magnéticos.

- Discos ópticos (CD y DVD): permiten un acceso aleatorio y en algunos casos la modificación de los datos. Su vida útil es mayor que en los casos anteriores, variando desde los cinco a diez años de los productos con menos calidad a varias décadas para los de mayor calidad.

- Mantenimiento, soporte y programas de reemplazo. Los componentes del sistema deben ser reemplazados cíclicamente cada dos años. Los 
elementos de hardware normalmente tienen una vida útil de en torno a los cinco años antes de que el soporte técnico pueda ser difícil de obtener. Los soportes de almacenamiento también necesitan una actualización regular (reescribir los datos) y un reemplazo periódico por nuevos soportes.

- Transferir los datos a nuevos soportes de forma periódica. Los sistemas de almacenamiento descansan en una segura y completa réplica de los datos más que en alargar la vida útil de los soportes. Los datos deben ser copiados de soporte a soporte para evitar el impacto del deterioro de los materiales. A medida que nuevos tipos de soportes prueban su utilidad para el almacenamiento, los datos deben ser transferidos desde los soportes más antiguos. Esto debe realizarse antes de que los componentes de hardware o software necesarios para acceder a los datos sean retirados.

- Establecer condiciones de almacenamiento y manejo apropiadas. Los soportes de almacenamiento digital deben ser almacenados en condiciones que no aceleren su ritmo de deterioro natural. El principal riesgo para los soportes es la excesiva humedad y temperatura, suciedad $\mathrm{u}$ otras particularidades que puedan entorpecer el acceso a los datos, y en el caso de soportes ópticos, la luz que puede dañar los datos inscritos. Las cintas magnéticas en la actualidad están protegidas de tal forma que el borrado accidental debido a su exposición a un campo magnético no suele ser un grave problema. Tales cintas pueden ser utilizadas como sistema de almacenamiento digital. Normalmente se las dispondrá en una sala acondicionada con una temperatura controlada y una humedad relativa establecidas entorno a los 18 grados centígrados y $40 \%$ respectivamente, un flujo continuo de aire limpio con una limpieza diaria que evite la acumulación de polvo y basura. Estas condiciones no deben fluctuar más de dos grados y un $10 \%$ de humedad relativa en un período de 24 horas. Los soportes ópticos tales como los CD deben ser almacenados en unas condiciones similares, incluyendo además una estancia semioscura para limitar su sensibilidad a la luz. Si bien se ha afirmado que las temperaturas extremadamente bajas, en torno a los cero grados, pueden ser perjudiciales para los soportes, esto no ha sido demostrado. 
Por otro lado, también pueden ayudar otros factores relativos al control del medio ambiente en que se almacena los objetos. Como básicos podríamos señalar:

- Mantener una temperatura en torno a $\operatorname{los} 20^{\circ} \mathrm{C}$.

- Mantener la humedad relativa en torno al 40\%.

- Evitar las oscilaciones rápidas o prolongadas de temperatura y humedad.

- Evitar la exposición a campos magnéticos y a fuentes de humos.

- Prohibir la comida y/o bebida en lugares de almacenamiento, así como el fumar.

- Almacenar los medios de forma vertical.

- Utilizar siempre las cajas originales.

- No abrir las cajas que protegen las cintas.

- Evitar la suciedad.

- Mantener los medios en sus cajas excepto cuando se estén usando.

- No tocar la superficie de los soportes, por ejemplo la cara grabable de los CD.

- Los CD deberían marcarse sólo en la parte superior y utilizando marcadores adecuados.

- No dejar los soportes en los aparatos de visualización cuando no se estén usando.

Políticas de redundancia y copias de seguridad: la importancia de la duplicación y las copias de seguridad no pueden ser desdeñadas, son fundamentales en todos los programas de conservación como un seguro básico contra el deterioro o pérdida si sólo existiera una única copia. Si bien almacenar múltiples copias de los mismos datos ofrece alguna protección contra los fallos, los programas de conservación deben también considerar el riesgo de una situación catastrófica tal como incendios, inundaciones, etc., que pueda dañar todas las copias existentes en un mismo lugar. Almacenar copias en diferentes lugares es un requisito básico.

Planificación contra los posibles desastres: deben diseñarse planes de recuperación de datos tras una situación de fallo que son estándares en las tecnologías de la información. Los planes deben incluir situaciones hipotéticas de recuperación de datos a partir de soportes dañados, pero la recuperación de 
datos es costosa e incierta y debería ser vista como una alternativa poco satisfactoria a la correcta recuperación a partir de copias de seguridad.

\section{9.- Bibliografía}

AGN. 2005. Política de archivos [en línea]. En: Compendio de políticas culturales. Bogotá: Ministerio de Cultura, pp. 325-344. ISBN 978-958-9177-33-4. [Consulta: 12 diciembre 2014]. Disponible en: http://www.archivogeneral.gov.co/politicas

Bonal Zazo, J. L. 2001. La descripciónarchivística normalizada. Origen, fundamentos, principios y técnicas. Gijón: Trea. ISBN: 84-9704-010-4

CIA. 2000. ISAD (G). Norma internacional de descripción archivística. $2^{\mathrm{a}}$ ed. Madrid:Subdirección General de Archivos Estatales. ISBN 84-369-3403-2

CIA. 2004. ISAAR (CPF) Norma internacional sobre los registros de autoridad de archivos relativos a instituciones, personas y familias. $2^{\mathrm{a}}$ ed. Madrid: Dirección General del Libro, Archivos y Bibliotecas. ISBN 84-8181-2 $11-0$

CIA. 2007. ISDF Norma internacional para la descripción de funciones. Madrid: Ministerio de Cultura.

CIA. 2008. SDIAH Norma internacionalpara describir instituciones que custodian fondos de archivo. París: Consejo Internacional de Archivos.

CONGRESO DE COLOMBIA. 2003. Ley 814 de 2003. Ley Cine. Bogotá: Diario Oficial.

Cruz Mundet, J. R. 2003. La gestión de los documentos electrónicos como función archivística [en línea]. AABADOM: Boletín de la Asociación Asturiana de Bibliotecarios, Archiveros, Documentalistas y Museólogos, 14 (2), 4-10. ISSN 1131-6764. [Consulta: 12 diciembre 2014]. Disponible en: https:// aabadom.files.wordpress.com/2009/10/75_0.pdf

DICCIONARIO. 1995. Diccionario de terminología archivística. $2^{a}$ ed.〔en línea]. Madrid: Ministerio de Educación, Cultura y Deporte. ISBN 84-8181-066-5. [Consulta: 28 septiembre 2014]. Disponible en: http://www.mecd.gob.es/cultura-mecd/areas-cultura/archivos/mc/dta/diccionario.html

Ecured. 2014. Conservación de documentos [en línea]. En: Ecured: Conocimiento con todos y para todos. [Consulta: 28 septiembre 2014]. Disponible en: http://www.ecured.cu/index.php/Conservaci\% C3\%B3n_de_documentos

Edmondson, R. 2004. Filosofía y principios de los archivos audiovisuales. París: UNESCO. [Consulta: 12 diciembre 2014]. Disponible en: http://unesdoc.unesco.org/images/0013/001364/136477s.pdf

Fundación Patrimonio Fílmico Colombiano. 2006. Historia de la Fundación Patrimonio Fílmico Colombiano. En: Fundación Patrimonio Fílmico Colombiano[en línea]. Bogotá. [Consulta: 25 septiembre 2014].Disponible en:http://www.patrimoniofilmico.org.co/index.php/informacion/ historia

Gómez Mascarell, S. 2012. Análisis de los elementos descriptivos aplicados en las plataformas de los archivos audiovisuales en el entorno de Internet. Una aproximación a las Normas UNE-EN 15477 y 15907 [en línea]. Tesis de máster. Valencia: Máster Universitario en Contenidos y Aspectos Legales en la Sociedad de la Información. [Consulta: 12 diciembre 2014]. Disponible en: http://riunet.upv.es/ handle/10251/27702 
HEREDIA HERRERA, A. 1991. Estado de la cuestión sobre instrumentos de descripción y normas de descripción. Irargi, 4, 183-205. ISSN 1130-9008. [Consulta: 12 diciembre 2014]. Disponible en:http://eah-ahe.org/pdf/revista4.es.pdf

HEREDIA HERRERA, A.1998.La Norma ISAD (G): análisis crítico [en línea]. Revista del Archivo General de la Nación, 18, 31-42. ISSN 259-2371. [Consulta: 12 diciembre 2014]. Disponible en: http://infolac2.ucol.mx/documentos/isad.pdf

MARQUES DE FONSECA, V. M. 2004. La Norma ISAAR (CPF). $2^{\text {a }}$ edición [en línea]. Ponencia con el mismo título, presentada en el I Congreso Nacional de Archivología en Brasilia, el 26 de noviembre de 2004. RAN:Revista del Archivo Nacional de costa Rica, 69(1-12), 43-50. [Consulta: 12 diciembre 2014]. Disponible en: www.archivonacional.go.cr

Ministerio de Tecnologías de la Información y las Comunicaciones. 2009. Guías cero papel en la administración: 3. Documentos electrónicos〔en línea].Bogotá: Imprenta Nacional. [Consulta: 12 diciembre 2014]. Disponible en: http://programa.gobiernoenlinea.gov.co/apc-aa-files/Cero_papel/ guia-3-documentos-electronicos-v 1.pdf

Ronco López, M.M. 2009. Los documentos audiovisuales y su conservación. En:Los servicios de documentación en los medios de comunicación del País Vasco. Bilbao, Universidad del País Vasco,pp. 73-100. ISBN 978-84-9860-202-9

SAINT LAURENT, G. 1998. CONSERVAPLAN Documentos para conservar, 8. El cuidado y manejo de grabaciones sonoras [en línea]. Caracas: Biblioteca Nacional de Venezuela. ISBN 980-319-144-6. [Consulta: 12 diciembre 2014]. Disponible en: http://www.bnv.gob.ve/pdf/Conser8.pdf

SOMEILLÁN LÓPEZ, M., A. GÓMEZ FERNÁNDEZ y G. GONZÁLEZ JUNCO 2006. Aspectos teóricos y conceptuales útiles para el diseño e implementación de una política de conservación preventiva [en línea]. Acimed,14(6). Disponible en: http://bvs.sld.cu/revistas/aci/vol14_6_06/ acio7606.htm. ISSN-e 2307-2113

Tadic, L. 2012.Video preservation for the Millennia [enlínea].AMIA Tech Review, 4.Hollywood: Association of Moving Image Archivist. [Consulta: 26 septiembre de 2014]. Disponible en: http:// www.amiatechreview.org/V12-05/papers/tadic.pdf. ISSN 2167-860X

UNESCO. 1980. Recomendación sobre la salvaguardia y la conservación de las imágenes en movimiento [en línea]. 27 de octubre de 1980. [Consulta: 26 agosto 2004]. Disponible en: http:// portal.unesco.org/es/ev.php-URL_ID=13139\&URL_DO=DO_TOPIC\&URL_SECTION=201.html

UNESCO. 2003a. Directrices para la preservación del patrimonio digital [en línea]. Camberra, Australia: Biblioteca Nacional de Australia. Marzo 2003. [Consulta: 26 agosto 2014]. Disponible en: http://unesdoc.unesco.org/images/0013/001300/130071s.pdf

UNESCO. 2003b. Carta sobre la preservación delpatrimonio digital [en línea]. París: UNESCO. 15 de octubre de 2003. [Consulta: 25 septiembre 2014]. Disponible en: http://portal.unesco.org/es/ev.phpURL_ID $=17721 \&$ URL_DO=DO_TOPIC\&URL_SECTION=201.html

UNESCO. 2013. Día Mundial del Patrimonio Audiovisual. En: Organización de las Naciones Unidas para la Educación, la Ciencia y la Cultura [en línea]. París: UNESCO. [Consulta: 25 septiembre 2014]. Disponible en: http://www.unesco.org/new/es/unesco/events/prizes-and-celebrations/ celebrations/international-days/world-day-for-audiovisual-heritage-2013/

UNIVERSAL. 2013.Miriam Garzón: la memoria fílmica de Colombia. En: El Universal [en línea]. Cartagena, Colombia. [Consulta: 25 septiembre 2014]. Disponible en: 
MEI, II, Vol. 5, no 9, pág. 155

http://www.eluniversal.com.co/suplementos/dominical/miriam-garzon-la-memoria-filmica-decolombia-1 10774

\section{0.- Notas}

1 Director del Programa de Sistemas de Información y Documentación. Universidad de La Salle. npulido@lasalle.edu.co

2 Directora Ejecutiva de la Red Audiovisual de Archivos de EEUU, fue profesora adjunta en la Universidad de Nueva York. Dicta conferencias en las áreas de gestión de activos digitales, audiovisuales, conservación digital y metadatos.

3 La presente normatividad recoge la citada en el texto: Documento técnico para la conformación de colecciones patrimoniales en la Dirección Archivo de Bogotá. (Inédito). Luis Enrique Rodríguez Baquero y Gustavo Adolfo Ramírez Ariza (Investigación y redacción), Alcaldía mayor de Bogotá D.C. Diciembre de 2010. Cap. I: "Fundamentos Normativos, Históricos y Técnicos sobre Patrimonio, Cultura y Archivos", pp. 11-16. Para el presente trabajo se citan los artículos que se consideran más pertinentes para el objeto de la metodología de valoración de archivos audiovisuales.

4. Se toman como documento electrónico teniendo en cuenta los criterios de creación, de origen y de forma y formato. 